\title{
Evolution of Respiratory tests in Children with Idiopathic Scoliosis Corrected by Posterior Approach
}

\author{
Acevedo, $\mathrm{I}^{\star}$, Dominguez $\mathrm{F}^{*}$, Burgos $\mathrm{J}^{\star *}$, Martín $\mathrm{J}^{\star}$ \\ *Ramon y Cajal Hospital, Dept of Anaesthesiology \& Intensive Care, Madrid, Spain, **Ramon y Cajal Hospital, \\ Traumatology Dept, Madrid, Spain.
}

\begin{abstract}
After surgical correction of thoracic scoliosis a greater cardiorespiratory adaptation to exercise would be expected from correction of the ribcage associated with deformity of the vertebral column. However, there is no clear evidence regarding whether said tolerance to maximum exercise improves in the medium term after surgery in patients with mild and moderate curves.
\end{abstract}

\begin{abstract}
Background and Goal:
Patients with idiopathic scoliosis have well documented decrease tolerance to maximum exercise. After surgical correction of thoracic scoliosis a greater cardiorespiratory adaptation to exercise would be expected, however, there is no clear evidence that the tolerance to maximum exercise improves in the medium term after surgery in patients with mild and moderate curves.
\end{abstract}

\begin{abstract}
Materials and Methods:
Twenty patients diagnosed with adolescent idiopathic scoliosis (AIS), ages 12 to 17 (mean 13) were studied. All curves were classified as Lenke $1 A$ and showed a mean magnitude of $60,3+9,9 \mathrm{Cobb}$ angle. The initial heart rate (HR INI), maximum heart rate (HR MAX), final heart rate (HR FIN), initial oxygen saturation (SAT O2 INI), final oxygen saturation (SAT O2 FIN), maximum oxygen consumption (VO2 MAX), production of $\mathrm{CO} 2$ (VCO2), ventilation and $\mathrm{CO} 2$ quotient (VE/CO2), respiratory exchange rate (RER), ventilator capacity at maximal exercise (VE MAX), and test duration were recorded before surgery and after 2 years of follow-up by a similar test of maximum tolerance to exercise on a treadmill following the standard Bruce protocol.
\end{abstract}

\section{Cobb angle/ RER changes}

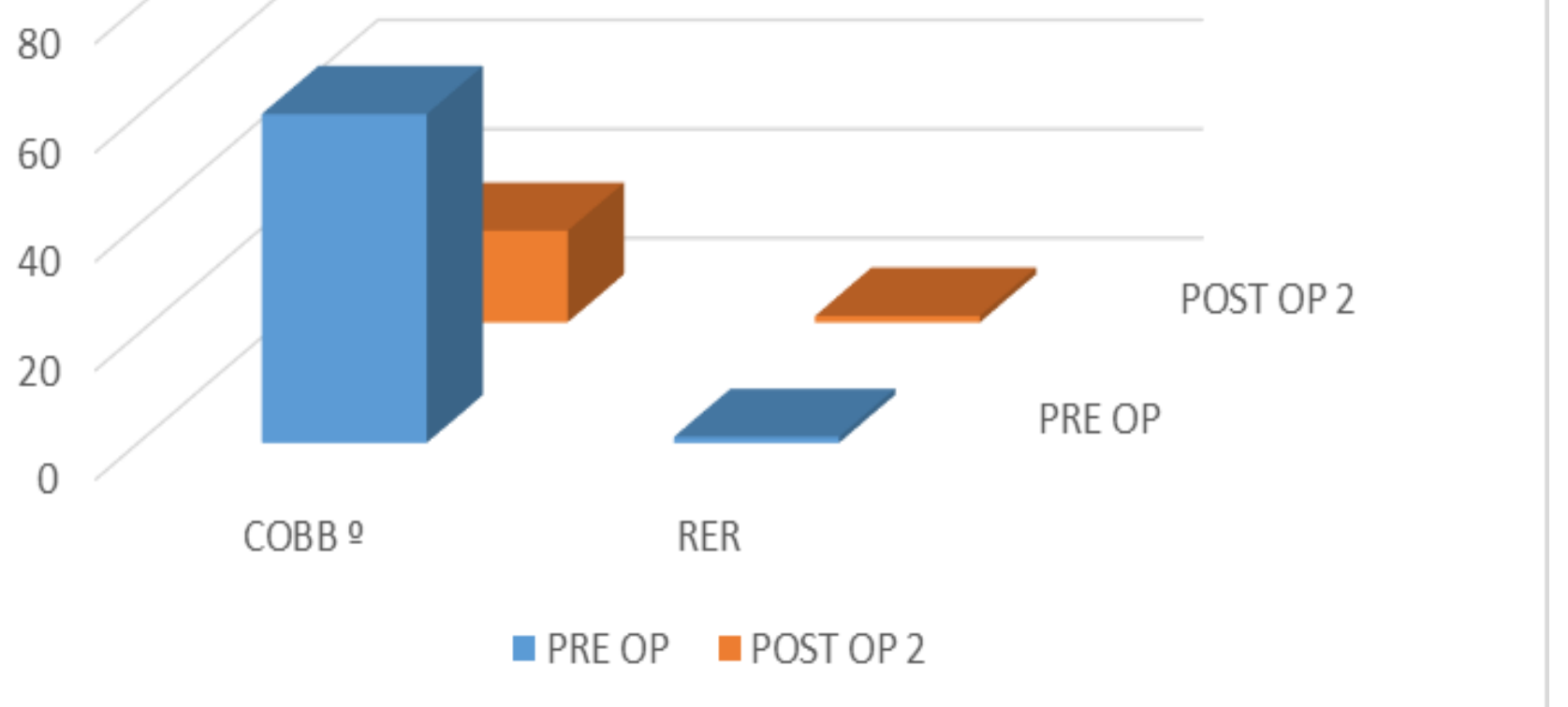

1.Jhonston, C. Richards, B. Sucato, C. Correlation of preoperative deformity magnitude and pulmonary function tests in adolescent idiopathic scoliosis. Spine 2011. 15;36(14):1096-102
Results and Discussion:

The curves averaged were $16,8+2,8$ Cobb 2 years after surgery. At 2 years after surgery, the patients showed similar values to the values measured in the preoperative period. There were only significant differences in RER $(1,12+0,09$ preop/ $1,20+0,12$ postop). Oxygen saturation at the end of the test was significantly decreased 2 years after surgery. In the post-operative test we can find a close correlation between duration and VE, which probably means better respiratory efficiency. Also was a close correlation between VO2 MAX, VE, degree of correction and final Cobb degree.

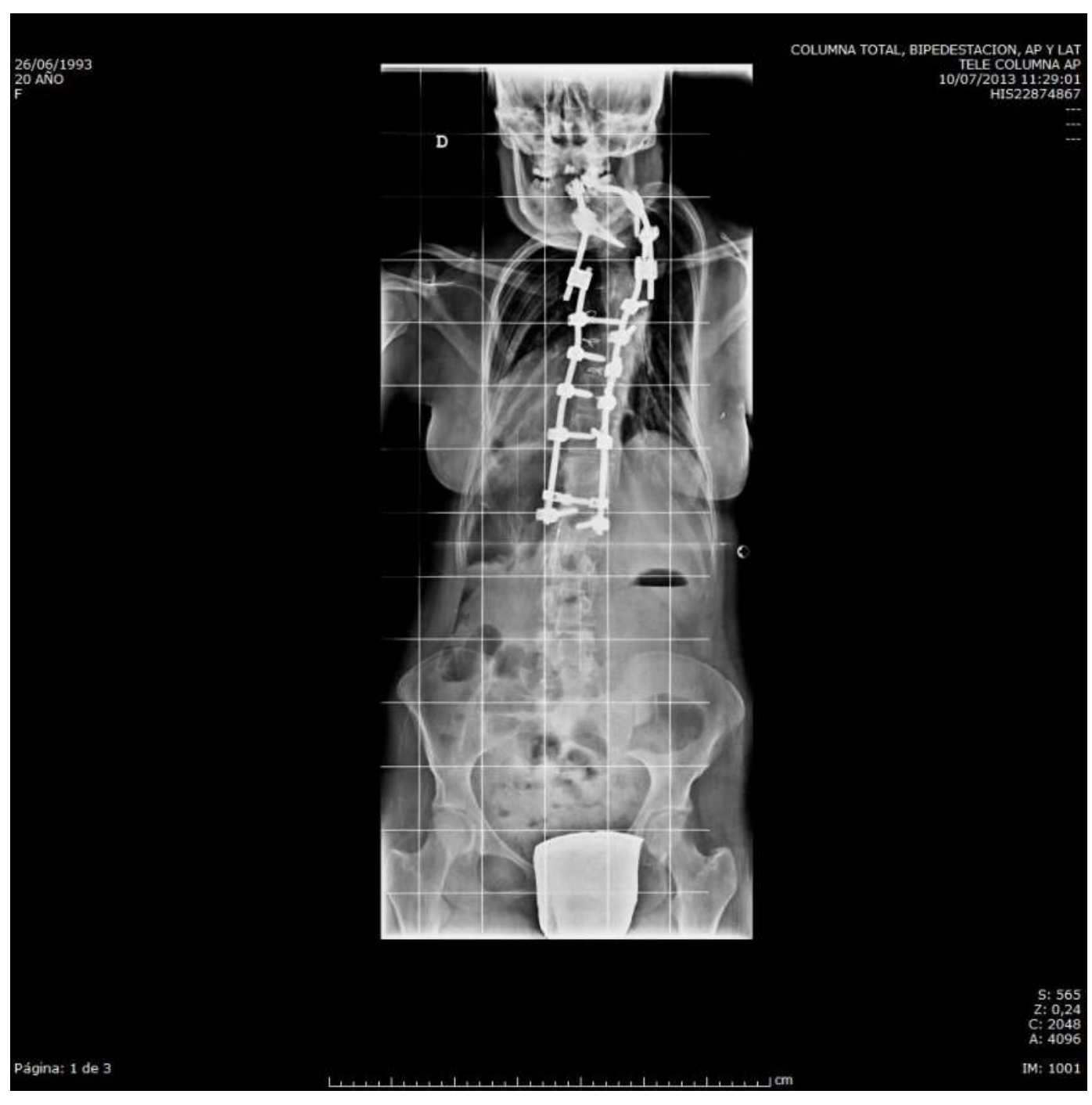

\section{Conclusion:}

Taken together, patients with moderate to severe AIS showed limited cardiorespiratory tolerance to maximum exercise that was no substantially modified two years after surgery, although the respiratory efficiency was improved by the improvement of many of the parameters. These findings suggest that the reduced cardiopulmonary function during exercise is not strictly associated with the deformity of the spine and can be due to and intrinsic factor such as muscle weakness and external factor such as the absence of physical conditioning in these kind of patients. 